\title{
Monolithic integration of vertical-cavity surface-emitting lasers with in-plane waveguides
}

\author{
Jeremy Witzens ${ }^{\text {a) }}$ and Axel Scherer \\ Department of Electrical Engineering 136-93, California Institute of Technology, \\ Pasadena, California 91125 \\ Gregory Pickrell, Duane Louderback, and Peter Guilfoyle \\ OptiComp Corporation, 215 Elks Point Road, Zephyr Cove, Nevada 89448
}

(Received 13 August 2004; accepted 18 January 2005; published online 2 March 2005)

\begin{abstract}
The ability to couple light from a vertical-cavity surface-emitting laser into a planar, on-chip waveguide creates new opportunities for achieving higher levels of integration and functionality. Here we propose to use a strong grating etched into a waveguide defined into the top layer of the epitaxially grown structure, so that epitaxial regrowth is not required. By introducing a defect mode into the cavity we were able to achieve a $40 \%$ coupling efficiency even though light is coupled through a ninety degrees bend. We also show that polarization control of the vertical-cavity surface-emitting laser is enhanced by coupling to the defect mode. Calculations were performed using the finite-difference time-domain method. (C) 2005 American Institute of Physics.
\end{abstract} [DOI: $10.1063 / 1.1880440]$

Coupling from vertical cavities to in-plane waveguides in a III-V material enables the monolithic integration of vertical-cavity surface-emitting lasers ${ }^{1}$ (VCSEL), $p-i-n$ photodetectors, and lithographically defined planar photonic devices in a single chip. For example, such a chip could be flip-chip bonded on a CMOS chip to implement optical clocking. Previously, VCSELs have been coupled to on-chip, planar waveguides by introducing a grating within the internal waveguide. ${ }^{2}$ However, it is advantageous to be able to define the grating coupler in the top layer of the structure rather than inside the vertical cavity because this removes the necessity for regrowth. In addition, this decouples the growth process from the lithography, so that a variety of planar devices can be subsequently etched into the top layer. However, coupling light through a ninety degrees bend with a strong external grating presents difficulties due to the fact that the Bragg condition of the grating also corresponds to its second-order bandgap. In previous designs where the grating coupler was inside the VCSEL cavity, the efficiency of the grating coupler was not an issue because noncoupled light stayed inside the cavity. However, an external grating coupler needs to have a high efficiency. Here these coupling difficulties are overcome by introducing a defect into the grating and defining a horizontal cavity.

We investigate with the finite-difference time-domain method (FDTD) a vertical cavity defined by a multilayer stack of alternating $\mathrm{GaAs}$ and $\mathrm{Al}_{0.866} \mathrm{GaAs} \lambda / 4$ films, where $\lambda=0.959 \mu \mathrm{m}$ is the lasing wavelength of the VCSEL. The refractive indeces of the thin films are $n_{\mathrm{GaAs}}=3.52$ and $n_{\mathrm{AlGaAs}}=3.02$. A defect is introduced in the thin film stack by increasing the thickness of one of the AlGaAs layers to $\lambda / 2 / n_{\text {AlGaAs }}$. Above the stack an $807 \mathrm{~nm}$ thick oxidized $\mathrm{Al}_{0.98} \mathrm{GaAs}$ buffer layer separates the top GaAs waveguiding layer from the VCSEL. The refractive index of the oxide $\left(n_{\text {oxide }}\right)$ is assumed to be 1.56 and the thickness of the top GaAs layer is $\lambda / 2 / n_{\text {GaAs }}(140 \mathrm{~nm})$. A grating is etched into

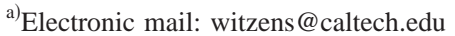

the top GaAs layer to transfer the light emitted by the VCSEL into the waveguide. The individual grates consist of $60 \mathrm{~nm}$ wide, $50 \mathrm{~nm}$ deep trenches. A defect is introduced in the grating coupler by locally changing the distance between two successive grates (the "grating defect" as opposed to the vertical-cavity defect). Finally, an $\mathrm{SiO}_{2}$ cladding layer is assumed to be deposited on top of the grating after etching, in order to symmetrize the refractive index around the GaAs waveguiding layer $\left(n_{\mathrm{SiO}_{2}}=1.46\right)$. The geometry of the device as well as the emitted $E$ field are shown in Fig. 1.

First, the grating is optimized in such a way as to maximize the coupling between the transverse electric (TE) mode of the waveguide ( $E$ field along the $z$ axis and $B$-field along the $y$ axis) and a Gaussian beam propagating normal to the waveguide ( $E$ field along the $z$ axis and $B$ field along the $x$

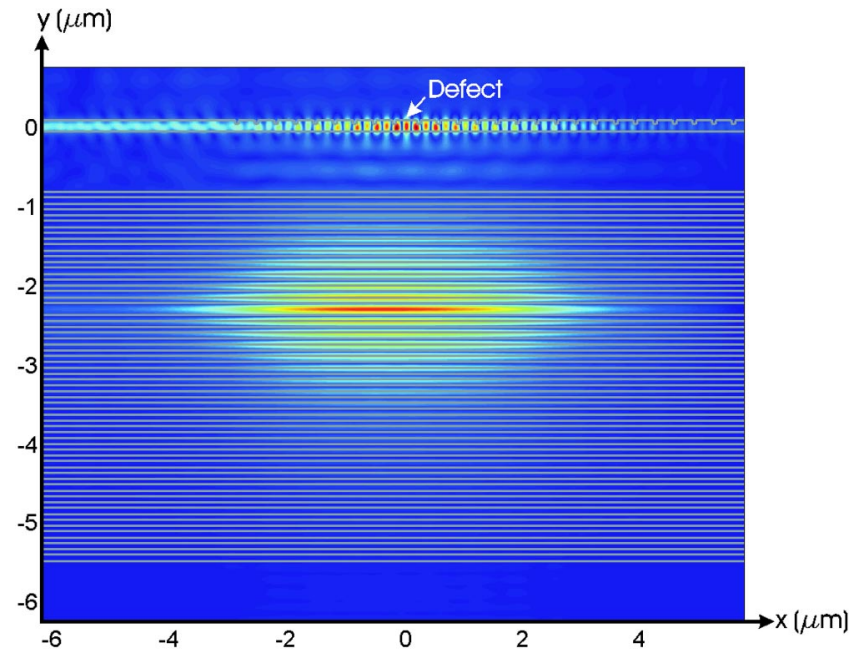

FIG. 1. (Color online) $E$ field $\left(\left|E_{z}\right|\right)$ in the vertical cavity and in the grating coupler (red corresponds to high intensity and blue to low intensity). The gray lines show interfaces between films of different refractive index. Alternating GaAs and AlGaAs layers define the VCSEL. Above the multilayer stack, an oxidized AlGaAs layer separates the waveguiding GaAs layer (with the grates) from the VCSEL. 
axis). The axes are defined in Fig. 1. Then the coupling between the grating defect and the vertical cavity is investigated. One of the limitations of the grating coupler is that it only couples TE polarized light, thus it is necessary to control the polarization of the VCSEL. The thickness of the AlGaAs oxide buffer layer determines the feedback from the grating defect into the vertical cavity. This feedback is used to enhance the quality factor $(\mathrm{Q})$ of the TE vertical-cavity mode. FDTD calculations are performed with a spatial resolution of $0.01 \mu \mathrm{m}$ and a time step of $0.005 \mu \mathrm{m}^{-1}$ (in units of $c_{0}=1$, where $c_{0}$ is the speed of light in vacuum).

First, we consider a defect-less, homogeneous grating, defined by etching trenches into the GaAs waveguiding layer. To outline the difficulties linked to the ninety degrees bend, it is convenient to consider the converse problem that consists in coupling from the waveguide into the vertical cavity. The Bragg condition to couple light out of the waveguide at a ninety degrees angle also corresponds to the second order bandgap of the grating, so that most of the power incoming from the waveguide is reflected back instead of coupled out. In this particular geometry, the effective index of the waveguide is $n_{\mathrm{WG}}=2.92$ and the grating period is $\Lambda=0.336 \mu \mathrm{m}$. In order to compensate for these reflections, a second mirror is needed to recycle them, i.e., we need a cavity in the waveguide plane. Thus we introduced a defect into the grating by increasing locally the distance between two successive grates. The number of grates on the right side of the defect is chosen to be very large ( 20 grates) so that almost no power is transmitted to that side of the cavity; the number of grates left of the defect are chosen so as to minimize reflections (9 grates). In order to efficiently couple into a Gaussian field the displacement has to be carefully chosen. Indeed the fractions of the grating before and after the defect may scatter the field out of the waveguide with different phases. However, in order to couple into a single lobe Gaussian beam, the fields extracted on the two sides of the defect have to be in phase. Here we form a defect by increasing the separation by $\lambda / 4 / n_{\mathrm{WG}}$, that corresponds to a defect mode with even $E_{z}$. Defects have been previously used in horizontal-cavity, surface emitting distributed feedback (DFB) lasers to obtain a single lobe far field radiation pattern. ${ }^{3}$

The definition of a defect inside the grating has the additional benefit of enhancing the overlap of the far field pattern of the grating with the VCSEL emission idealized as a Gaussian beam (Fig. 2). Indeed the field intensity has a naturally "humped" shape around the defect that has a bigger overlap with a Gaussian beam than the decaying exponential obtained with homogeneous gratings. Usually, the field overlap with a Gaussian is enhanced by varying the scattering strength of the grates throughout the grating. ${ }^{4}$ However, we found this to be impractical in the case of a 90 degrees coupling angle. Due to the strong back-reflections induced by the grating, the field penetration into the grating is very short. In order to compensate for this, we chose the grates as weak as we estimated reasonable for standard fabrication techniques $(60 \mathrm{~nm}$ wide with a very shallow etch), leaving no degree of freedom for additional tailoring. We calculated the overlap of the grating far-field pattern $4 \mu \mathrm{m}$ away from the waveguide with Gaussian beams of varying width (vectorial calculation) and found that the best overlap was with a Gaussian with a full width at half maximum (FWHM) of $3.1 \mu \mathrm{m}$ (93\% overlap).

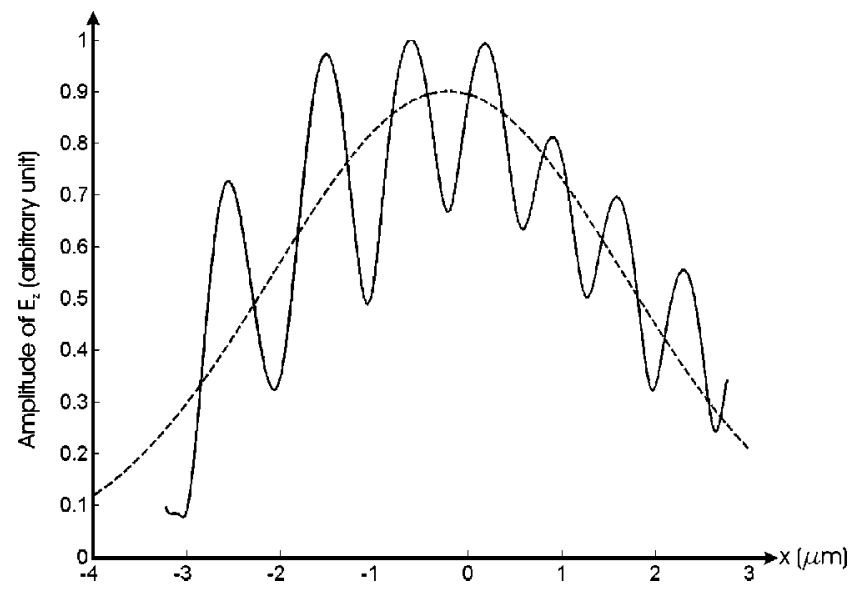

FIG. 2. Amplitude of $E_{z}$, inside the oxidized AlGaAs cladding layer $4 \mu \mathrm{m}$ below the stand-alone grating coupler (continuous line). The best Gaussian fit $(\mathrm{FWHM}=3.1 \mu \mathrm{m})$ is also shown (dashed line).

Figure 3 shows the efficiency of the stand alone coupler over a range of wavelengths. By stand alone we mean that the coupler is not combined with the vertical cavity, but merely clad by $\mathrm{SiO}_{2}$ on the patterned side and $\mathrm{AlGaAs}$ oxide on the other side. In such a configuration, we found that the extracted field had the same intensity and profile in the $+y$ and $-y$ directions, even though the structure is slightly asymmetric (this may be linked to the fact that the thickness of the waveguiding GaAs layer is chosen to be $\lambda / 2 / n_{\mathrm{GaAs}}$ and has a resonance in the $y$ direction). In Fig. 3, we plotted the sum of the power extracted to $+y$ and $-y$, or equivalently two times the power extracted towards $-y$. Due to the resonant nature of the coupling mechanism, the pass-band is quite small compared to nonresonant grating couplers, but it is still wider than the line width of a VCSEL. The coupling efficiency of the stand-alone grating coupler is $46 \%$ in each direction.

Next, we investigate the interaction between the grating defect and the VCSEL cavity. When the Gaussian beam is coupled from the oxidized AlGaAs layer into the waveguide, there is also a strong grating induced back-reflection $(26 \%){ }^{5}$

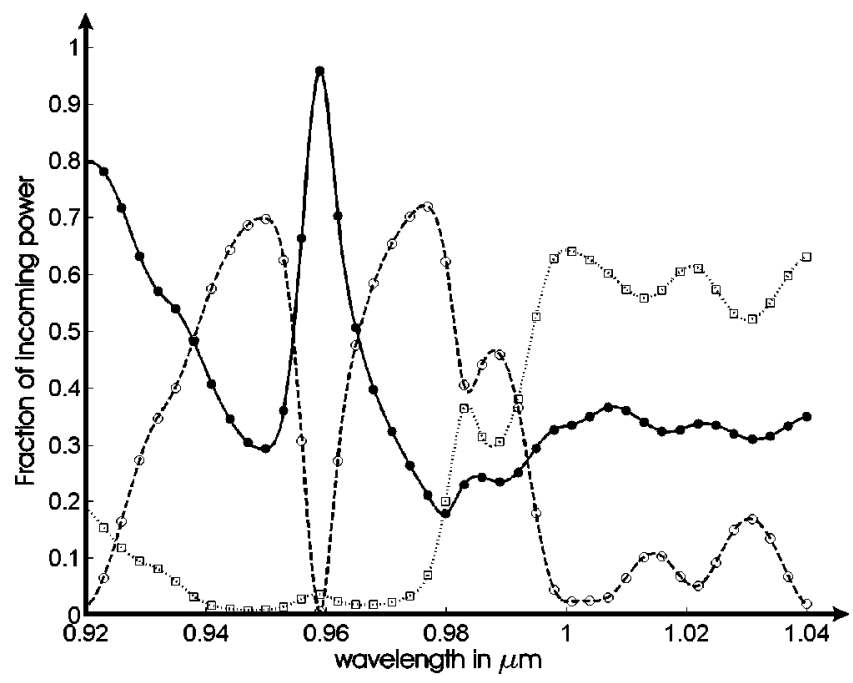

FIG. 3. Coupling efficiency (dots), back-reflection into the waveguide (circles) and transmission to the other side of the grating (squares) as a function of wavelength. This data corresponds to a stand-alone grating coupler. The plotted coupling efficiency is the sum of the coupling efficiency in the $+y$ and in the $-y$ directions. 
The reflected power is not lost but recycled inside the VCSEL cavity, thus increasing the $Q$ factor of the VCSEL provided the thickness of the oxidized AlGaAs layer is chosen so that the reflection adds constructively. Also the reflection induced by the grating has a different phase than the reflection from a plain dielectric interface, so that the thickness of the AlGaAs oxide layer has to be modified from $\lambda / 4 / n_{\text {oxide }}$ to compensate for the extra phase. In the final design we obtained a $40 \%$ coupling efficiency from the vertical cavity to the waveguide.

The TM-mode of the waveguide ( $B$ field along the $z$ axis) has a different effective index than the TE-mode $(E$ field along the $z$ axis), so that the TM-polarization is not resonant at $\lambda=0.959 \mu \mathrm{m}$. As a consequence, the strong grating-induced reflection is much weaker for TM-polarized vertical-cavity modes $(8 \%)$. This can be used to enhance polarization control inside the VCSEL by tailoring the vertical-cavity $Q$ factors of the TE and the TM polarizations. In particular, the presence of the grating has only a small effect on the $Q$ factor of the TM-polarization, while the $Q$ factor of the TE-polarization is strongly enhanced. We simulated a device with 19 layers stacked between the verticalcavity defect and the oxidized AlGaAs layer (10 layers of GaAs and 9 layers of AlGaAs, interleaved). This cavity had a $Q$ factor of 450 without the grating coupler. After the unpatterned waveguiding layer was added, the $Q$ factor of the TE-mode stayed roughly unchanged $(Q=555)$ due to the fact that this layer has a resonance in the $y$ direction (thickness $\left.\lambda / 2 / n_{\mathrm{GaAs}}\right)$. When the grating was included, the $Q$ factor of the TE-mode was enhanced to 1520 while the $Q$ factor of the TM mode stayed quite low (600). The enhancement of the $Q$ factor of the TE-mode is slightly more than what would be expected from the $26 \%$ reflection (1400). This might be due to a folded cavity effect (i.e., the effective pathlength of the cavity is enhanced). On the other hand the enhancement of the $Q$ factor of the TM-mode is lower than what would be expected from the $8 \%$ reflection (800). This is due to the fact that the reflection adds a different phase increment to the TM field than to the TE field, so that the AlGaAs oxide layer does not have the correct thickness to optimally enhance the $Q$ factor of the TM-mode.

The relative enhancement of the $Q$ factor of the TEmode will facilitate polarization control in the VCSEL and might remove the need for more complex polarization splitting grating couplers. ${ }^{6}$ The strong reflection induced by a grating coupler defined on top of a VCSEL has been previously used to reduce the $Q$ factor of one of the modes for polarization-control, but in that case the goal was not to outcouple into a waveguide and the $Q$ factor of the gratingcoupled mode was reduced rather than enhanced. ${ }^{7}$

Figure 4 shows a fabricated structure. The VCSEL is defined by selective hydrogen implantation and gain guiding. An aperture is left open in the electrode at the location of the

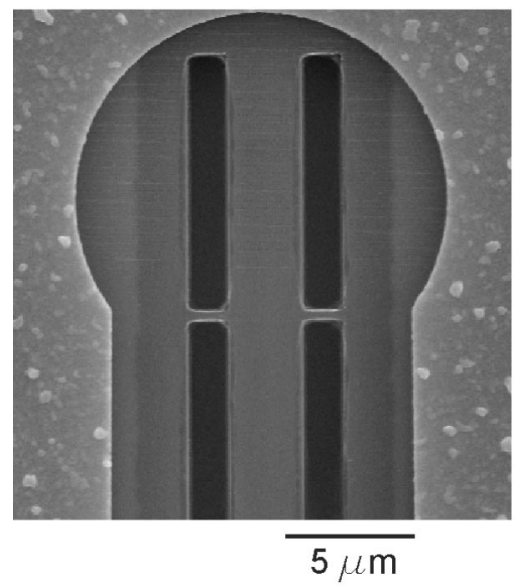

FIG. 4. Scanning electron micrograph of a fabricated structure.

VCSEL (circular aperture) as well as around the waveguide. Trenches etched into the GaAs layer define the (segmented) waveguide. The trenches are interrupted to prevent the waveguide peeling off. In order to electrically pump the VCSEL, the AlGaAs buffer layer is only selectively oxidized through the trenches (the oxidized region can be recognized as the shaded region around the waveguide). The grating is etched above the VCSEL within the circular aperature, however the grates are not resolved in this micrograph. The image was taken before deposition of the top $\mathrm{SiO}_{2}$ cladding layer.

In conclusion, we have shown theoretically that by introducing a defect into a grating coupler light can be efficiently coupled around a ninety degrees bend. We obtained $40 \%$ coupling efficiency from a vertical cavity to an in-plane waveguide defined in the topmost layer of the same chip. Furthermore, feedback from the defect mode of the grating coupler to the vertical cavity enhances polarization-control of the VCSEL.

The authors would like to acknowledge generous support from the AFOSR program "optical logic with gain" under contract number F49620-03-1-0418.

${ }^{1}$ J. L. Jewell, S. L. McCall, Y. H. Lee, A. Scherer, A. C. Gossard, and J. H. English, Appl. Phys. Lett. 54, 1400 (1989).

${ }^{2}$ D. A. Louderback, G. W. Pickrell, H. C. Lin, M. A. Fish, J. J. Hindi, and P. S. Guilfoyle, Electron. Lett. 40, 1064 (2004).

${ }^{3}$ S. Li, G. Witjaksono, S. Macomber, and D. Botez, IEEE J. Sel. Top. Quantum Electron. 9, 1153 (2003).

${ }^{4}$ K. A. Bates, L. Li, R. L. Roncone, and J. Burke, Appl. Opt. 32, 2112 (1993).

${ }^{5}$ G. A. Golubenko, A. S. Svakhin, V. A. Sychugov, and A. V. Tischchenko, Sov. J. Quantum Electron. 15, 886 (1985).

${ }^{6}$ D. Taillaert, H. Chong, P. I. Borel, L. H. Frandsen, R. M. De La Rue, and R. Baets, IEEE Photonics Technol. Lett. 15, 1249 (2003).

${ }^{7}$ M. Abdou, F. Pigeon, A. V. Tishchenko, O. Parriaux, Y. Ouerdane, S. Reynaud, J.-C. Pommier, and H.-J. Fuchs, IEEE J. Quantum Electron. 39, 614 (2003). 